\title{
Mass spectrometry-based proteomic exploration of the human immune system : focus on the inflammasome, global protein secretion, and T cells
}

\section{Nyman, Tuula A.}

2017

Nyman , T A , Lorey , M B , Cypryk , W \& Matikainen , S 2017 , ' Mass spectrometry-based proteomic exploration of the human immune system : focus on the inflammasome, global protein secretion, and T cells ' , Expert review of proteomics. , vol. 14 , no. 5 , pp. 395-407 . https://doi.org/10.1080/1

http://hdl.handle.net/10138/234525

https://doi.org/10.1080/14789450.2017.1319768

acceptedVersion

Downloaded from Helda, University of Helsinki institutional repository.

This is an electronic reprint of the original article.

This reprint may differ from the original in pagination and typographic detail.

Please cite the original version. 


\section{Mass spectrometry-based proteomic exploration of the human immune system: focus on the inflammasome, global protein secretion, and T cells}

\section{Tuula A Nyman, Martina B Lorey, Wojciech Cypryk \& Sampsa Matikainen}

To cite this article: Tuula A Nyman, Martina B Lorey, Wojciech Cypryk \& Sampsa Matikainen (2017): Mass spectrometry-based proteomic exploration of the human immune system: focus on the inflammasome, global protein secretion, and T cells, Expert Review of Proteomics, DOI: 10.1080/14789450.2017.1319768

To link to this article: http://dx.doi.org/10.1080/14789450.2017.1319768

Accepted author version posted online: 13

Apr 2017.

Submit your article to this journal $[\pi$

Џll Article views: 2

Q View related articles $₫$

View Crossmark data $\nearrow$ 
Publisher: Taylor \& Francis

Journal: Expert Review of Proteomics

DOI: $10.1080 / 14789450.2017 .1319768$

Mass spectrometry-based proteomic exploration of the human immune system -focus on the inflammasome, global protein secretion, and $T$ cells

Tuula A Nyman ${ }^{1 *}$, Martina B Lorey², Wojciech Cypryk ${ }^{3}$ and Sampsa Matikainen ${ }^{2}$

1. Department of Immunology, Institute of Clinical Medicine, University of Oslo and Rikshospitalet Oslo, Oslo, Norway

2. University of Helsinki and Helsinki University Hospital, Rheumatology, Helsinki, Finland

3. Department of Bioorganic Chemistry, Center of Molecular and Macromolecular Studies, Lodz, Poland

\section{Corresponding author}

Tuula Nyman

Department of Immunology, Faculty of Medicine,

University of Oslo

Sognsvannsveien 20, Rikshospitalet, 0372 OSLO,

Norway

E-mail: tuula.nyman@medisin.uio.no

Key words: immune system, mass spectrometry, inflammasome, protein secretion, innate immunity, $\mathrm{T}$ cell 


\section{ABSTRACT}

Introduction: The immune system is our defense system against microbial infections and tissue injury, and understanding how it works in detail is essential for developing drugs for different diseases. Mass spectrometry-based proteomics can provide indepth information on the molecular mechanisms involved in immune responses. Areas covered: Summarized are the key immunology findings obtained with MSbased proteomics in the past five years, with a focus on inflammasome activation, global protein secretion, mucosal immunology, immunopeptidome and T cells. Special focus is on extracellular vesicle-mediated protein secretion and its role in immune responses.

Expert commentary: Proteomics is an essential part of modern omics-scale immunology research. To date, MS-based proteomics has been used in immunology to study protein expression levels, their subcellular localization, secretion, posttranslational modifications, and interactions in immune cells upon activation by different stimuli. These studies have made major contributions to understanding the molecular mechanisms involved in innate and adaptive immune responses. New developments in proteomics offer constantly novel possibilities for exploring the immune system. Examples of these techniques include mass cytometry and different MS-based imaging approaches which can be widely used in immunology. 


\section{Introduction}

Pathogens can rapidly adapt and evolve and thereby avoid detection by the human immune system. However, multiple defense mechanisms have developed to recognize and eliminate pathogens, including innate and adaptive cell-mediated immunity (Figure 1). The innate immune system is the first line of defense against microbial infections. It also responds to host factors that arise during tissue damage and metabolic dysregulation. Inflammasomes are multimeric cytosolic protein complexes that mediate innate immune responses to microbial infection, cellular damage, and metabolic dysregulation [1]. The assembly of inflammasomes triggers activation of inflammatory cysteine protease caspase-1 and proteolytic processing and secretion of pro-inflammatory cytokines Interleukin (IL)-1 and IL-18. These cytokines are important mediators of inflammatory responses and are critical in both local and systemic inflammation. Activation of innate immunity is essential to control infections and provide the necessary signals to trigger adaptive immunity [1]

Activation of adaptive immunity is usually required to completely eradicate microbial infections.

Protein secretion is an important part of the immune response. Proteins can be secreted through multiple pathways (summarized in Fig 2). According to the Human Protein Atlas [2], approximately $39 \%$ of the $\sim 20,000$ human protein-coding genes are 
predicted either to express a signal peptide that is required for secretion through the ER/Golgi secretory pathway or to have at least one transmembrane region, suggesting active transport of the corresponding protein out of the cell. Proteins that lack a signal peptide can be secreted through unconventional, vesicle-mediated pathways. Immune cells secrete a wide range of proteins including immunomodulatory factors (e.g., cytokines and chemokines) that are indispensable for proper coordination of appropriate cellular responses.

Mass spectrometry (MS)-based proteomics can provide in-depth information about how the immune system is regulated and the molecular mechanisms involved in immune responses. The main goal of this review is to summarize the key findings in molecular immunology in the past five years using different MS-based proteomics approaches, with a focus on inflammasome activation, global protein secretion and extracellular vesicles, mucosal immunology, immunopeptidome and T cells. Key findings obtained with proteomics studies related to these topics are summarized in Table 1.

\section{Proteomics contributes to immunology research at multiple levels}

Modern MS-based proteomics methods give detailed spatio-temporal information on proteins on a global scale. Proteomics provides information on protein expression, sub-cellular localization, post-translational modifications (PTMs), and interactions. Most proteomics methods use a so-called 'bottom up' approach, where the proteins are first digested into peptides; the resulting peptides are analyzed by liquid chromatography (LC)-tandem mass spectrometry (MS/MS) followed by computational data analysis. Several methods are available for the identification and 
quantification of thousands of proteins from biological samples and comparison of the proteome profiles of different samples (reviewed in $[3,4]$ ). Quantitative MS-based proteomics provides data on protein expression levels and also on protein localization and trafficking inside the cells when it is combined with sub-cellular fractionation. Several studies have used this approach to characterize changes in sub-cellular proteomes due to viral infection $[5,6]$ and other stimuli activating innate immune responses $[7,8]$. In addition to global screening, MS-based proteomics provides methods for targeted protein quantification (reviewed in [9]).

Protein phosphorylation is a widely studied PTM with major impacts on most cellular signaling cascades. In phosphoproteomics, the phosphorylated peptides need to be enriched before the LC-MS/MS analysis. The two most common enrichment methods take advantage of titanium dioxide or immobilized metal ion affinity chromatography. Advances in phosphopeptide enrichment methods and MS analysis, together with improved data analysis tools, have made it possible to identify thousands of phosphoproteins from cellular samples (reviewed in $[10,11])$. Phosphoproteome studies on host responses to viral infection [12-16] have shown that viral infection alters the phosphorylation status of hundreds of proteins involved in pathways critical to the host response to infection. For example, phosphoproteome characterization of influenza $A$ virus (IAV) infection in human macrophages, combined with bioinformatics and functional studies, showed that cyclin-dependent kinases are activated upon IAV infection; targeting these kinases with small-molecule inhibitors could be a novel strategy to treat severe influenza virus infections [13]. This study also highlights the importance of using primary cells in proteome-level studies to obtain the most novel and biologically meaningful data for further functional studies. 
In addition to large-scale phosphoproteomic analysis, MS-based studies focusing on the detailed characterization of individual protein's phosphorylation status have contributed to our understanding of immune system regulation. Very recently, Lee et al. used this approach to show that infection-specific phosphorylation of glutamylprolyl tRNA synthetase induces antiviral immunity [17]. In a very elegant study, Liu and co-workers used targeted quantification by MS to demonstrate that the adaptor molecules of the innate immunity, MAVS, STING, and TRIF are phosphorylated to mediate activation of transcription factor interferon regulatory factor 3 [18].

Proteins act in cells in complexes with other proteins, and in-depth knowledge of these complexes is essential to understand cellular signaling in detail. Affinity purification (AP) combined with MS is a widely-used strategy to characterize protein complexes. Several methods of AP are available and have been reviewed recently [19]. The AP-MS methods include traditional immunoprecipitation using a protein of interest as bait. The main drawback of this technically simple approach is the high background of proteins non-specifically binding to the antibody. Therefore, many approaches have been developed using two-step AP to maximize the recovery of specific interactions. AP-MS has produced significant new knowledge of key proteins involved in immune responses [20-24]. These include studies of 14-3-3 and Rab GTPase proteins. 14-3-3 proteins are a family of conserved regulatory molecules that can bind a multitude of functionally diverse signaling proteins through phosphorylation-dependent interactions. Öhman et al. [21] combined phosphoproteomics with quantitative 14-3-3 protein AP to characterize the 14-3-3 protein-mediated signaling pathways activated during cytosolic dsRNA-induced 
innate immune responses in human keratinocytes. Through extensive bioinformatics analysis of the combined datasets combined with functional studies, they showed that sirtuin 1 and Rel A-associated inhibitor are novel regulators of antiviral innate immune responses. Rab GTPases regulate many stages of membrane traffic, including vesicle formation, vesicle movement, and membrane fusion. In a recent study, Li et al. [24] depicted the interactomic landscapes of major mammalian Rab GTPase family proteins in dendritic cells (DCs) and provided a global view of intracellular membrane organization in combination with AP-MS and imaging tools. Further analysis showed that the Rab32 subnetwork of proteins has anti-microbial functions.

\section{Intracellular proteome characterization of inflammasome activation}

Inflammasomes are critical components of the innate immune system that activate inflammation and contribute to the initiation and pathology of human disease in many ways. Inflammasomes are critical for the clearance of pathogens and damaged cells, but overwhelming inflammasome activation is a major driver of autoimmune disease and metabolic disorders [1]. The canonical inflammasome protein complexes consist of caspase-1, adapter protein apoptosis-associated speck-like protein containing a caspase-recruitment domain (ASC), and a sensor protein. These sensor proteins belong to NOD-like receptor (NLR) or to the absent in melanoma 2 (AIM2)-like receptor families and include NLRP1, NLRP3, NLRC4, and AIM2 inflammasomes[1]. Of these, NLRP3 inflammasome is the most extensively studied because of its activity in many human diseases, including autoinflammatory diseases, Alzheimer's disease, atherosclerosis, and diabetes [25]. 
Several AP-MS-based studies have identified novel components and regulators of inflammasomes. A systematic proteomic screen for proteins that associate with DNA led to the identification of the AIM-2 inflammasome, which is involved in the cytoplasmic recognition of double-stranded DNA [26]. In addition, AP-MS resulted in the identification of end-binding protein 1 as a crucial component of the AIM-2 inflammasome [27]. Further, He and co-workers recently identified an essential mediator of NLRP3 activation called NIMA Related Kinase 7 (NEK7) using AP-MS [28]. NEK7 associates with NLRP3 following ATP stimulation and is required for NLRP3-mediated caspase-1 activation [28]. Imiquimod is a small-molecule ligand of Toll-like receptor-7 that is licensed for the treatment of viral infections and skin cancers. It is also a known activator of NLRP3 inflammasome in myeloid cells [29]. A recent study used AP-MS with a bead-coupled imidazoquinoline to identify the targets of imiquimod [30]. These results show that imiquimod inhibits the quinone oxidoreductases NQO2 and mitochondrial Complex I. This resulted in reactive oxygen species formation and thiol oxidation and was followed by NLRP3 activation via NEK7. AP-MS-based techniques have also been exploited to study the posttranslational regulation of inflammasome activation: Yan et al. showed that dopamine inhibits NLRP3 inflammasome activation via a second messenger cyclic adenosine monophosphate that binds to NLRP3 and promotes its ubiquitination and degradation via the E3 ubiquitin ligase MARCH7 [31].

In addition to canonical inflammasomes, non-canonical inflammasomes have recently been described [32]. Non-canonical caspase-4/5 inflammasome activates pyroptosis, an inflammatory form of cell death in response to infections of gram-negative bacteria [62]. Human non-canonical caspase-4/5 inflammasome can also activate the 
canonical NLRP3 inflammasome by an unidentified mechanism [33]. The AP-MS approach identified a new component of the inflammasomes called gasdermin D [34]. Gasdermin D is required for pyroptosis in response to both NLRP3 inflammasome and non-canonical inflammasome activation [25].

Quantitative proteomics has also produced new knowledge on the inflammasomes. Worah and co-workers utilized label-free quantitative MS to identify differences between the proteome profiles of primary human DC subsets. They showed that plasmacytoid DCs do not express caspase-1, the central component of canonical inflammasomes, and that they also express other inflammasome-related proteins at low levels [35]. This suggests that the role of plasmacytoid DCs is not related to inflammasome-mediated secretion of pro-inflammatory cytokines: instead, they are potent producers of antiviral type I interferons [36].

\section{Secretome analysis of immune cells}

Protein secretion through multiple pathways is an important part of immune responses. In immunology, studies in protein secretion have focused mostly on analyzing secretion of cytokines and chemokines using antibody-based assays such as ELISA. However, recent system-level characterizations using MS-based proteomics approaches have shown that immune cells activate a much more global protein secretion than just secretion of cytokines and chemokines. The global pattern of secreted proteins (secretome) of a cell depends largely on its activation state; the detailed characterization of secretomes provides valuable information for understanding immune response mechanisms. At present, high-resolution MS combined with advanced sample preparation methods allows for analysis of the total 
secretome from low numbers of cells: Meissner and co-workers were able to achieve low picogram sensitivity by quantifying the time-resolved release of 775 proteins from as little as 150,000 stimulated mouse macrophages per condition [37]. This study linked specific secretory profiles to the activation of distinct intracellular signaling adaptor proteins and demonstrated that the secretions of many pro-inflammatory mediators have redundant mechanisms, leading to a potentially vast increase in their secretion.

Total secretomes of human cells upon virus infection have been widely studied with the rationale of understanding the cellular response to the infection, elucidating the physiopathology of the resulting disease as well, and determining potential therapeutic targets. Global secretome analysis of human macrophages after infection by IAV [5] and herpes simplex virus [38] revealed massive release of danger signal proteins and identified secreted host factors that have a role in antiviral defense. In addition to viral infection, the secretomes of human macrophages have been studied following different stimuli [39-41]. These include ATP, monosodium urate (MSU) and -glucans. Extracellular ATP and MSU are endogenous danger signals known to activate inflammatory responses; $\beta$-glucans are the main constituents of fungal cell walls, triggering an effective innate immune response. The secreted proteins identified in these studies involved many danger signal proteins that amplify inflammatory response during innate immune activation, such as annexins, high mobility group proteins and S100 and heat shock proteins. Bioinformatic characterization of these secretomes revealed that most identified proteins did not have the signal sequence required for classical ER/Golgi-mediated secretion and that 
most of the proteins are found in the ExoCarta database, having been identified in the exosomes of multiple organisms [42].

The effects of bacterial infection on the secretome of several human cell types have also been investigated with proteomic tools. For example, total secretome analysis revealed strong differences in the inflammatory responses induced by different Staphylococcus aureus strains [43]. Uhlmann and colleagues identified a novel secreted streptococcal factor that can potentially trigger neutrophil activation and degranulation during Streptococcus pyogenes infections of human neutrophils [44]. In addition to live bacteria, secretome analysis has been performed in cells that have been activated with lipopolysaccharide (LPS), the cell wall component of gramnegative bacteria. Secretome analysis of endothelial cells stimulated with extracellular LPS to activate Toll-like receptor (TLR)4 led to the discovery of 19 potential biomarkers for sepsis [45]. Most secreted proteins induced by LPS stimulation were related to the regulation of actin cytoskeleton [45].

Secretome analysis of human adipose tissue-derived mesenchymal stem cells (hASCs) revealed secretion of several chemokines and cytokines in response to stimulation with tumor necrosis factor (TNF), a key pro-inflammatory cytokine [46]. This demonstrated that TNF-treated hASCs secrete factors that drive the monocyte migration and subsequent tissue regeneration. Oh and co-workers performed secretome analysis of human monocytes from newborn and elderly donors stimulated with several commonly used adjuvants [47]. These adjuvants activate an innate immune response, typically via TLR stimulation. TLR-mediated immune responses show distinct differences between different age groups, and the study 
demonstrated that adjuvants induce different yet partially overlapping secretomes that vary with the adjuvant types and the age of the study participants. These data are important when designing vaccines for certain age groups.

\section{Proteome analysis has shown that extracellular vesicles play an important}

\section{role in immune responses}

Proteins that do not carry an N-terminal signal peptide required for the classical ER/Golgi secretory pathway are released unconventionally. They are packed into various types of membrane-enclosed structures, collectively called "extracellular vesicles" (EVs) [48]. These EVs include microvesicles (MVs) that bud directly from the plasma membrane and are shed into the extracellular space, exosomes that are formed when multivesicular bodies fuse with the plasma membrane, and other vesicles whose origin has been linked with different cellular processes including secretory autophagy, apoptosis and lysosome secretion. EVs are released from virtually all cell types for long-distance intercellular communication, carrying a diverse and well-protected cargo of biomolecules: proteins, nucleic acids, and bioactive lipids. They can travel considerable distances throughout human body fluids and tissues and deliver molecular information to recipient cells upon endocytosis. When released from stimulated cells, they have been shown to modulate the immune response [49].

Proteomics has been used extensively for the characterization of EVs released from immune cells. For proteomics analysis, EVs are isolated from a multitude of biological fluids and growth media using different protocols involving high-speed centrifugation, affinity purification, precipitation, and filtration-based methods. For example, Groot Kormelink and co-workers used high-speed ultracentrifugation to isolate EVs from 
mast cells following immunoglobulin E-mediated cell activation. They established that during their degranulation mast cells release EVs containing mast cell-specific proteases and concluded that these EVs and their content are potentially important immune regulators [50]. Another proteomic study utilizing similar EV isolation method showed that foam cells, which are fat-laden macrophages in atherosclerotic plaques, secrete more EVs than unstimulated macrophages. These foam cell-derived EVs promote vascular smooth muscle cell migration and adhesion and potentially play a role in the disease progression [51]. The optimal methods for isolation and separation of different EVs are still highly debated [52]. A recent study by Kowal and colleagues provided an extensive characterization of proteins in different EV classes secreted from human DCs. They first purified different EVs by their sedimentation speed and then either by their behavior upon upward floatation into iodixanol gradients or by immuno-isolation. Based on quantitative proteome analysis of separated EV populations, they suggest novel protein markers to distinguish different EV populations [53].

\subsection{EVs secreted during microbial infection}

Proteomic analyses of EVs released from virally infected cells have been performed with the purpose of understanding the observed overlap between natural endocytosis-exocytosis pathways and the virus life cycle. Accumulating evidence for viral hijacking of human EV secretory pathways and membranes raised the hypothesis of the existence of "Trojan exosomes," as carriers of viral genetic material, facilitating viral infections [54]. Consequently, identification of EV proteins directly associated with virus dissemination is of interest for the identification of novel drug targets and development of antiviral therapies. 
Several groups applied proteomics to analyze the EVs from human immunodeficiency virus (HIV)-1-infected cells. Li and colleagues revealed that EVs released from $\mathrm{HIV}$-1-infected $\mathrm{H} 9$ cells contain a unique and quantitatively different protein signature than exosomes released from uninfected cells and harbor regulatory molecules that impact the processes of cellular apoptosis and proliferation [55]. This study also showed that EVs facilitate the transfer of HIV-1 and viral constituents from infected macrophages to neighboring uninfected cells [56]. Meckes et al. performed a large-scale quantitative proteomic study, infecting eleven B cell lines with Kaposi sarcoma-associated virus and Epstein-Barr virus or with both viruses and purifying the secreted EVs. This study demonstrated that viral infection causes virus- and host cell-specific modifications of the proteome of secreted EVs that is correlated with and dependent on the expression of viral oncogene latent membrane protein-1. This discovery not only shed new light on the roles of EVs in the pathogenesis of these common viruses but also identified immune regulatory pathways affected by the EVs that directly facilitate virus survival and spreading [57]. By applying an SILAC quantitative proteomics approach, Zhao and colleagues revealed that hepatitis $B$ virus also alters the proteomic composition of hepatic cell line Huh-7-derived EVs [58]. A similar modulation was observed in the case of human T cell Tymphotropic virus-infected T cell lines [59].

A recent article described the proteomic characterization of EVs released from human macrophages upon IAV infection [60], showing a robust EV-mediated protein secretion as early as $9 \mathrm{~h}$ post-infection. Proteins secreted in response to IAV infection included many proteins involved in translation, such as components of 
spliceosome machinery and the ribosome. The data also shows that EVs derived from IAV-infected macrophages contain antiviral cytokines, fatty acid-binding proteins, copper metabolism Murr-1 domain proteins, and autophagy-related proteins. These data suggest an important role for EVs in intercellular communication during IAV infection.

Some of the most extensively studied EVs in the context of host cell-bacteria interaction involve those released from Mycobacterium tuberculosis-infected human macrophages. Hare et al. demonstrated that EVs from infected macrophages contain several interferon-inducible proteins [61]. Wang et al. compared the proteomic composition of EVs from Mycobacterium avium-infected macrophage-like THP1 cells. M. avium infection significantly changed the protein composition of exosomes isolated from THP-1 cells [62]. Exosomes isolated from infected cells contained components that induced immune responses in resting cells, suggesting an important function for exosomes in anti-mycobacterial host defense.

In addition to viral and bacterial infections, the role of fungal infection on EV secretion and composition has also been studied. Cypryk and co-workers showed that the major components of fungal cell walls, $\beta$-glucans, enhance vesicle-mediated protein secretion in human macrophages. The unconventionally secreted proteins included several receptors, such as cation-dependent mannose-6-phosphate receptor, macrophage scavenger receptor, P2X7 receptor, and several integrins [63]. A recent study of EVs from C. albicans-infected THP1 cells indicated that fungal stimulation induces secretion of EVs with alternated content of several signaling proteins 
including chitinase-3-like protein 1, which has been proposed as the component responsible for the pro-inflammatory properties of the secreted EVs [64].

\subsection{Inflammasomes and EV secretion}

The NLRP3 inflammasome activates caspase-1 and the subsequent secretion of proinflammatory cytokines in response to microbial infection and endogenous danger signals. A study utilizing iTRAQ-labelling-based quantitative proteomics linked caspase-1 to unconventional protein secretion as early as 2008 [65]. Subsequent proteomics studies showed that NLRP3 activators including ATP, -glucans, and monosodium urate activate robust unconventional vesicle-mediated protein secretion in human macrophages [39-41,63]. In contrast to these inflammasome activators, LPS-induced TLR4 triggering did not result in EV-mediated protein secretion despite the fact that TLR4 signaling resulted in a strong activation of gene expression [41]. Active forms of the lysosomal proteases cathepsins were identified in EVs after NLRP3 inflammasome activation $[40,41]$. Cathepsins are proteases that are required for NLRP3 inflammasome activation. This suggests that EVs released during inflammasome activation can amplify and/or activate NLRP3 inflammasome in recipient cells. In line with these results, Zhang and co-workers have shown that nigericin, a known activator of the NLRP3 inflammasome, activates EV-mediated protein secretion from LPS-primed macrophages [66]. They identified several Toll-like receptors, as well as components of the NF- B and NLRP3 inflammasome signaling pathways, in EVs isolated from LPS-primed and nigericin-activated mouse macrophages. These results also suggest that $\mathrm{EVs}$ isolated from macrophages after NLRP3 inflammasome activation can enhance inflammatory responses in recipient cells. 
A recent study showed that, in addition to NLRP3 inflammasome, also the noncanonical caspase-4/5 inflammasome can activate unconventional vesicle-mediated protein secretion in human macrophages [67]. The proteins secreted through EVmediated pathway contained ribosomal and danger signal proteins, including TLR4ligands S100A8 and prothymosin- $\alpha$ which may contribute to endotoxic shock during non-canonical inflammasome activation.

\section{Proteomics to study mucosal immunology}

Mucosal immunology is in the front-line status within the immune system preventing the uptake of pathogens and other foreign materials. Proteomics has been used to study mucosal specimens during microbial infection [68]. A label-free quantitative MS was used to study specimen isolated from small intestine of patients with acute and convalescent stages of Vibrio cholerae infection. This study identified 27 host proteins that were differentially abundant between the acute and convalescent stages of infection. The majority of these proteins had known roles in innate immunity including cytokine production and apoptosis. The authors conclude that a strong inflammatory response is generated in the gut mucosa early after onset of $V$. cholerae infection, which may be critical for the development of long-term immunity against $V$. cholerae [68].

Proteomics has also been used to identify disease mechanisms and novel biomarkers and from mucosal colon biopsies for inflammatory bowel disease (IBD) including Crohn's disease (CD) and ulcerative colitis (UC) [69-73]. Accurate differentiation between new onset $C D$ and $U C$ is demanding and therefore novel 
biomarkers are needed. Similarly, differentiation of $C D$ and intestinal tuberculosis (ITB) is challenging. Starr et al. used SILAC to analyze mucosal biopsies from 99 pediatric control and biopsies of patients with $C D$ and UC. The study identified two panels of candidate biomarkers for the diagnosis of IBD and the differentiation of IBD subtypes [71]. Rukmangadachar and co-workers analyzed colonic biopsies from inflamed mucosa of treatment-naïve patients with ITB, CD and controls with ITRAQ. This study concludes that there are differentially expressed proteins in tissue proteome of $C D$ and ITB but fails to identify biomarkers that could be used to differentiate these diseases. Bennike and co-workers analyzed mucosal colon biopsies from non-inflamed tissue of patients with UC and compared the proteomes of those samples with the samples taken from healthy controls with label-free quantitative MS-based proteomics. Proteins with increased abundances in the UC colon biopsies included proteins associated with neutrophil extracellular traps and proteins functioning in innate immunity [71]. These results suggest a role for innate immunity in the etiology of UC. Mottawea et al. found altered host proteome in newonset pediatric patients with DC [73]: especially mitochondrial proteins implicated in $\mathrm{H}_{2} \mathrm{~S}$ detoxification were expressed at low level. At the same the relative abundance of $\mathrm{H}_{2} \mathrm{~S}$ microbial producers was increased and dysfunctional host mitochondrial function was observed. The study shows that host-microbiota interactions are disturbed in $C D$ and provides a new mechanistic explanation for the pathogenesis of CD [73].

\section{Proteomic characterization of the immunopeptidome}

The immunopeptidome is the collection of peptides associated with and presented by major histocompatibility complex (MHC) molecules. Characterization of these 
peptides is important for the development of better vaccines and immunotherapies against autoimmunity. The MHC immunopeptidome is highly complex and MS-based approaches have proven to be the method of choice in these studies (reviewed in [74]). At present, most immunopeptidome studies utilize affinity column coupled with monoclonal antibody (mAb) specific for a certain MHC class or allotype followed by MS-analysis of the captured peptides.

Several recent studies have shown that it is possible to identify thousands of HLAbound antigenic peptides from diverse biological samples using this approach. The samples include celiac disease-associated MHC molecules, different cancer cell lines, fibroblasts, DCs, macrophages, and bronchoalveolar lavage (BAL) from patients with sarcoidosis [75-79].

PTMs are an additional source of complexity in the immunopeptidomes. So far, phosphorylated and arginine methylated HLA-bound peptides have been identified $[80,81]$. These modifications potentially provide a unique source of disease-related MHC peptides that can elicit specific immune responses which may offer a novel targets for immunotherapy.

The analyses of immunopeptidomes have usually been done using large amount of starting material not feasible to obtain from clinical samples. Heyder et al. optimized the method to identify HLA-DR-bound peptides from low cell numbers and utilized it to study BAL cells obtained from patients with sarcoidosis [78], and showed that the investigation of the BAL immunopeptidome from individual patients and healthy controls is possible with MS-based methods in order to identify disease-associated peptides. Moreover, a very recent report by Bassani-Sternberg et al. showed that advanced MS can be used directly for identification of mutated peptide ligands isolated from HLAs on the surface of native tumor tissues and concluded that these 
mutated peptide ligands yield true neoepitopes with high relevance for immunotherapeutic strategies in cancer [82].

The proteasome generates the epitopes presented on HLA class I molecules that elicit CD8+ T cell responses. The key step for the transformation of a protein into an HLA-I-restricted epitope is usually processed by the proteasome, which cuts proteins into peptides; alternatively, the proteasome can also cut and paste peptide sequences, thereby releasing peptide antigens that do not correspond to the original protein sequence. However, this proteasome-catalyzed peptide splicing has long been considered to occur only rarely. Liepe et al. developed an MS-based strategy to analyze this and unexpectedly revealed that a large fraction of HLA class I ligands are proteasome-generated spliced peptides [83]. Such merged peptides might turn out to be useful in vaccine or cancer immunotherapy development.

\section{Proteomic studies on T cells}

T cells play a central role in adaptive, cell-mediated immunity; their action is usually required for the final eradication of a microbial infection. Proteomics has been applied extensively to characterize T cells. Early quantitative MS-based proteomics studies on human primary CD4+ T helper (Th) cells revealed proteome changes in both microsomal and nuclear fractions [84-86]. These include the regulation of several immune-related proteins, including galectin-1, small GTPases GIMAP1 and GIMAP4, and SATB1, during Th cell differentiation. Recently, the proteomes of human Th1 and Th1/Th17 clones derived from intestinal biopsies of Crohn's disease patients were characterized using high-resolution MS [87]. In total, more than 7,000 proteins from the Th1 and Th1/Th17 clones were identified, with 334 proteins being differentially 
expressed. Major differences were observed for cytotoxic proteins that were overrepresented in the Th1 clones.

CD8+ cytotoxic T cells (CTLs) have also been characterized using quantitative proteomics $[88,89]$. Hukelmann et al. used high-resolution MS to map the proteome of CTLs and quantify the regulatory effect of selective inhibition of the mammalian target of rapamycin complex 1 (mTORC1) and combined inhibition of mTORC1 and mTORC2 on CTL proteomes. They identified almost 7,000 proteins from CTLs, demonstrating the diversity of the CTL proteome and how mTOR inhibitors control T cell function and program T cell signal-transduction pathways [88]. Böttcher et al. used transcriptome and proteome profiling of different memory $\mathrm{CD} 8^{+}$cell populations to demonstrate that $\mathrm{CX} 3 \mathrm{CR} 1$ is superior for classification of these cell populations [89]. This analysis allowed the authors to establish a core gene and protein signature shared by memory $\mathrm{CD}^{+}$cells with a cytotoxic function independent of their tissue localization. The results will help establish better immune monitoring that will improve guidance of immune therapies.

Navarro et al. used targeted proteomics, namely, selected reaction monitoring (SRM), to quantify PKD2 abundance in naïve CD8+ T cells [90]. PKD2 is a serine and threonine kinase that is activated in T cells by diacylglycerol and protein kinase $\mathrm{C}$ in response to stimulation of the $\mathrm{T}$ cell receptor $(\mathrm{TCR})$ by an antigen. They quantified the activation of PKD2 at the single-cell level and found that this kinase acts as a sensitive digital amplifier of TCR engagement. SRM can yield absolute quantification of target peptides from a particular protein in a complex sample. In principle, it also has the potential to detect proteins in the low-attomole range of abundance. 
Several phosphoproteome studies on T cell lines have aimed to dissect IL-2- and IL15-induced cell signaling events. In these studies, Osinalde and co-workers utilized SILAC labeling and high resolution MS combined with phosphotyrosine immunoprecipitation [91-93] and TiO2 enrichment of the phosphopeptides $[94,95]$ to demonstrate that the signaling pathways activated by IL-2 and IL-15 are very similar [94,95]. In addition, they combined antibody- and TiO2-based enrichment with SILAC labeling before MS analysis to show that serine/threonine phosphorylation of the scaffold protein Gab2 is an important step in regulating IL-2 signaling [96] Quantitative phosphoproteomics has also been used to characterize CD8+ CTLs in detail $[97,98]$. Ross and co-workers showed that CTLs contain an IL-2-induced JANUS kinase independent signaling network. This SRC family tyrosine kinasecontrolled signaling network regulates $10 \%$ of the CTL phosphoproteome and critically contributes to CTL function [97].

In addition to protein phosphorylation, TCR-induced ubiquitination is known to regulate the function of $\mathrm{T}$ cells. More specifically, the function of $\mathrm{T}$ cells is negatively regulated by the E3 ubiquitin-protein ligases CBL and CBLB. Voisinne et al. utilized AP-MS to analyze the dynamics of the CBL and CBLB signaling complexes formed after TCR stimulation [99]. They identified several proteins that had not yet been implicated in those signaling complexes and demonstrated that the CD5 transmembrane receptor constitutes a key scaffold for ubiquitin-ligase mediated ubiquitylation following TCR engagement [99]. These results provide a molecular basis for understanding the negative regulation of TCR signaling. 
Cluster of Differentiation 28 (CD28) is a protein expressed on T cells that provides co-stimulatory signals required for T cell activation and survival. Roncagalli et al. combined AP-MS analysis and mouse functional genomics to determine the mode of action of RLTPR cytosolic protein, also known as CARMIL2, which is essential for CD28 co-stimulation in mouse T cells. They developed mice that bear a genetic tag allowing for AP-MS analysis of the RLTPR interactome in primary T cells, showed that RLTPR acts as a scaffold, bridging CD28 to the CARD11/CARMA1 cytosolic adaptor and to the NF-KB signaling pathway, and identified previously unknown proteins in the CD28 signaling pathway [100].

\section{Expert commentary}

Proteomics is an essential part of modern life science research. Proteome-level information is needed to elucidate disease mechanisms, find new drug targets, and develop personalized medicines. To date, MS-based proteomics has been used in immunology to study protein expression levels, their subcellular localization, posttranslational modifications, and interactions in immune cells upon activation by different stimuli. These studies have contributed significantly to our understanding on the molecular mechanisms involved in both innate and adaptive immune responses (Table 1).

Post-translational modifications affect protein activity; sub-cellular localization also has a key impact on understanding the molecular mechanisms involved in immune responses in detail. Information about PTMs is also important for drug discovery. So far, most of the focus has been on phosphoproteome analysis, mainly because the techniques for these studies are already well established. However, the importance of other modifications is being increasingly appreciated, and previously 
uncharacterized modifications are frequently being discovered. In innate immunity, the cross-talk between protein phosphorylation and ubiquitination is an emerging topic $[101,102]$. Other, less well-studied PTMs that modulate innate immunity are acetylation, glutamylation, and deamidation [102]. A main challenge for PTM analyses is that they usually require that the modified peptides are enriched from the total peptide pool before MS-analysis, and enrichment methods for differentPTMs are newly established. Additionally, MS data analysis is more challenging than the analysis required for protein identification and quantification. However, the methods for both of these are actively being developed; it is likely that many new tools to facilitate these analyses will arise in the next five years.

Global proteome studies of secretomes and EVs have shown that unconventional, vesicle-mediated protein secretion is an essential part of immune responses. Most proteome studies of protein secretion have focused on protein identification and quantification. In addition to intracellular PTMs, there are some reports on protein modifications in EVs; it will be important to characterize these PTMs from EV proteins in more detail in the future $[103,104]$. Proteome studies have also highlighted the fact that vesicle-mediated protein secretion is activated upon canonical NLRP3 and noncanonical caspase-4/5 inflammasome activation. Future proteome studies are needed to reveal novel components and regulators of the non-canonical caspase- $4 / 5$ inflammasome.

An important proteomic technique to elucidate intracellular signaling pathways is APMS. This method has revealed several critical components and regulators of the inflammasomes. In AP-MS, the typical workflow includes one- or two-step affinity 
purification of the protein complex followed by MS-based identification and quantification of proteins in the complex. The starting material is most often the total cellular lysate; the method requires that false-positive interactions are minimized as efficiently as possible, both during sample preparation and in data analysis after MSanalysis.

Additionally, several targeted MS-based approaches have been developed that allow for MS-based monitoring of a set of pre-selected proteins from complex mixtures. In these methods, the proteins of interest do not need to be purified from the sample; the selection of the proteins to be measured and quantified is performed in the MS workflow. These methods can be used in immunology to complement traditional antibody-based assays such as western blotting and ELISA. The main advantages of these assays are that they are not dependent on the available antibodies and that they can be fully automated.

\section{Five-year view}

MS-based proteomics has made major contributions to understanding the molecular mechanisms involved in immune responses. New developments in proteomics constantly provide novel possibilities for advanced proteome analysis. Examples of novel proteomics techniques that will most likely be used widely in proteomics include mass cytometry and different MS-based imaging approaches. Mass cytometry enables high-dimensional, single-cell analysis of cell type and state. It combines the cellular analysis principles of traditional fluorescence-based flow cytometry with the 
selectivity and quantitative power of inductively coupled plasma-mass spectrometry; multiplexing of up to 40 independent measurements on a single cell is possible $[105,106]$.

Creating proteome maps is exemplified by a study of Marakalala et al.: they combined laser-capture microdissection, high resolution MS, and confocal microscopy to generate detailed molecular maps of human granulomas that are the pathological hallmark of tuberculosis [107]. They showed that the centers of granulomas have a pro-inflammatory environment characterized by the presence of antimicrobial peptides, reactive oxygen species and pro-inflammatory eicosanoids; conversely, the tissue surrounding the caseum has a comparatively anti-inflammatory signature. Based on the protein and lipid snapshots obtained, they hypothesize that the pathologic response to $M$. tuberculosis is shaped by the precise anatomical localization of these inflammatory pathways during the development of the granuloma.

The proteome of blood immune cells has been extensively investigated; however, so far, there is little data on tissue-resident immune cells. Very recently, Holzlechner et al. [108] characterized these cells in colon tissue, which exhibited a strong infiltration of immune cells. They used MALDI MS-imaging (MALDI MSI) and showed for the first time that it is well-suited for visualizing the spatial distribution of immune cells in human colon tissue. The MALDI MSI technique has vast potential for use in rapid investigations of tissue-specific features of cells in the future.

At present, the fundamental importance of proteomics in all life science research is well recognized. The participants of the National Institutes of Health Workshop in 
Clinical Proteomics in 2012 concluded that 'deeper understanding of the human proteome could help fill in the gaps between genomes and phenotypes, transform the way we develop diagnostics and therapeutics, and thereby enhance overall biomedical research and future healthcare' [109]. Additionally, the current methods allow for deeper and faster proteome characterization than what has ever been possible before. However, proteomics is still not routinely used in most immunology research groups. This is due to two main reasons: proteomics techniques are not always easily accessible to biomedical researchers, and more education is needed for biomedical students and researchers so they understand how proteomics could contribute to their research. As early as 2008 , one of the leading pioneers in MSbased proteomics, professor Matthias Mann, argued that the true integration of proteomics technology into molecular biology laboratories could be a paradigm shift for all of biology and biomedicine, but quantitative proteome measurements need to become as accessible and convenient as western blots are now for this to happen; equally important, proteomics should be incorporated into biology education [110]. Another key issue for implementing proteomics into immunology and other biomedical research is the seamless collaboration of researchers from multiple disciplines (immunology, proteomics, and bioinformatics).

\section{Key issues}

- Proteomics can provide detailed information on cellular signaling mechanisms involved in immune responses; MS-based proteomics has made important contributions to understanding the human immune system in more detail

- Developments in MS-based proteomics during the last decade have enabled deeper and more rapid proteome analysis than what has previously been 
possible; with current methods, it is possible to identify, quantify, and characterize thousands of proteins in a single experiment

- MS-based proteomics techniques have been applied extensively to characterize $T$ cells and immunopeptidomes

- Phosphoproteomics has shown that the functions of key receptors and adaptor molecules of the innate immune system are regulated by phosphorylation

- Proteomics has revealed several novel components and regulators of inflammasomes

- Global protein secretion analyses have shown that vesicle-mediated protein secretion plays an important role in innate immune responses

- Proteomics techniques develop constantly and MS-based imaging methods will offer important new possibilities for immunological studies

- To make full use of the power of proteomic techniques, true integration of this technique into immunology laboratories and close collaboration between immunology, proteomics and bioinformatics researchers are needed

\section{Funding}

The authors have received support from the University of Oslo and the University of Helsinki.

\section{Declaration of interest}

The authors have no relevant affiliations or financial involvement with any organization or entity with a financial interest in or financial conflict with the subject matter or materials discussed in the manuscript. This includes 
employment, consultancies, honoraria, stock ownership or options, expert testimony, grants or patents received or pending, or royalties.

\section{Figure legends}

Figure 1. Innate immunity is required for the activation of adaptive T cell-mediated immunity. Macrophages and dendritic cells (DCs) are central effector cells of innate immunity; their pattern recognition receptors (PRRs) detect the presence of pathogens and endogenous danger signals. This recognition results in the activation of innate immune cells. Macrophages and DCs start to secrete cytokines to mount an inflammatory response, chemokines to recruit other immune cells to the site of infection or inflammation, and other proteins inducing anti-microbial defense and tissue regeneration. Activation of inflammasomes, which are PRRs expressed by macrophages and DCs, triggers the secretion of IL-1 family cytokines and unconventional protein secretion in general. Activated innate immune cells also express co-stimulatory molecules, including cluster of differentiation (CD) 80 and CD86, on their surface which makes antigen presentation to T cells possible. Antigens are presented to T cells through the human leukocyte antigen (HLA) system, which is a gene complex encoding the major histocompatibility complex (MHC) proteins in humans. HLAs corresponding to MHC class I present peptides from inside the cell. This typically occurs during viral infections, resulting in the activation of cytotoxic T cells that kill virusinfected cells. HLAs corresponding to MHC class II present antigens from outside of the innate immune cell to T cells. These particular antigens stimulate $\mathrm{T}$ helper cell activation, which in turn stimulates B cell antibody production against the specific antigen. 

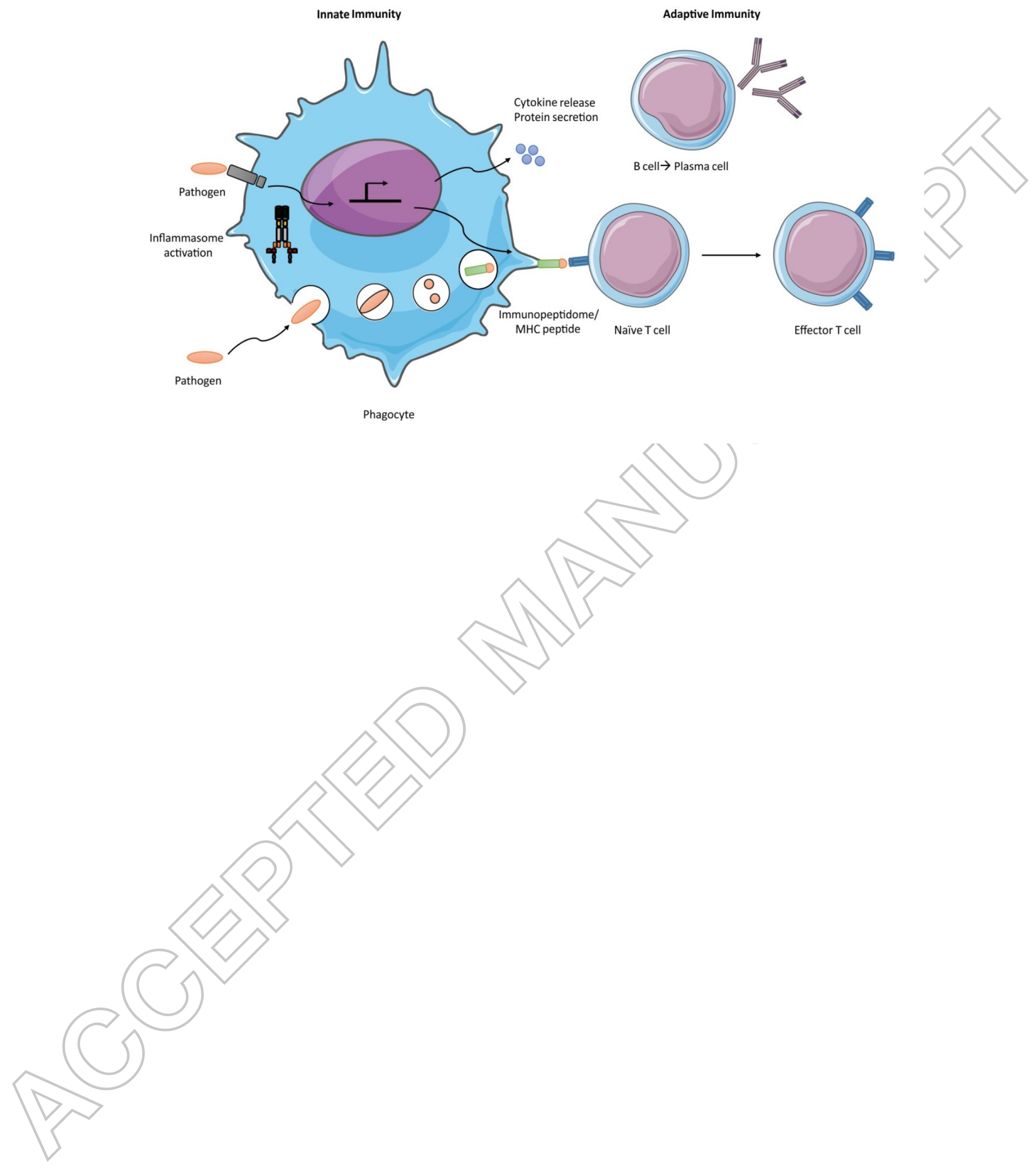
Figure 2. Protein secretion mechanisms in immune cells. A fraction of the secreted proteins contain an $\mathrm{N}$-terminal signal sequence and are secreted by the classical (ER/Golgi) secretory route. However, most of the proteins are secreted by unconventional means: an ATP-dependent $A B C$ transporter-assisted route, incorporation into extracellular vesicles (secretory lysosomes, exosomes and microvesicles) or bypassing Golgi. Additional mechanisms or combinations of different pathways of secretion probably exist, as signal sequence-containing proteins are also abundantly found on EVs. Figure adopted with permission from: [111]

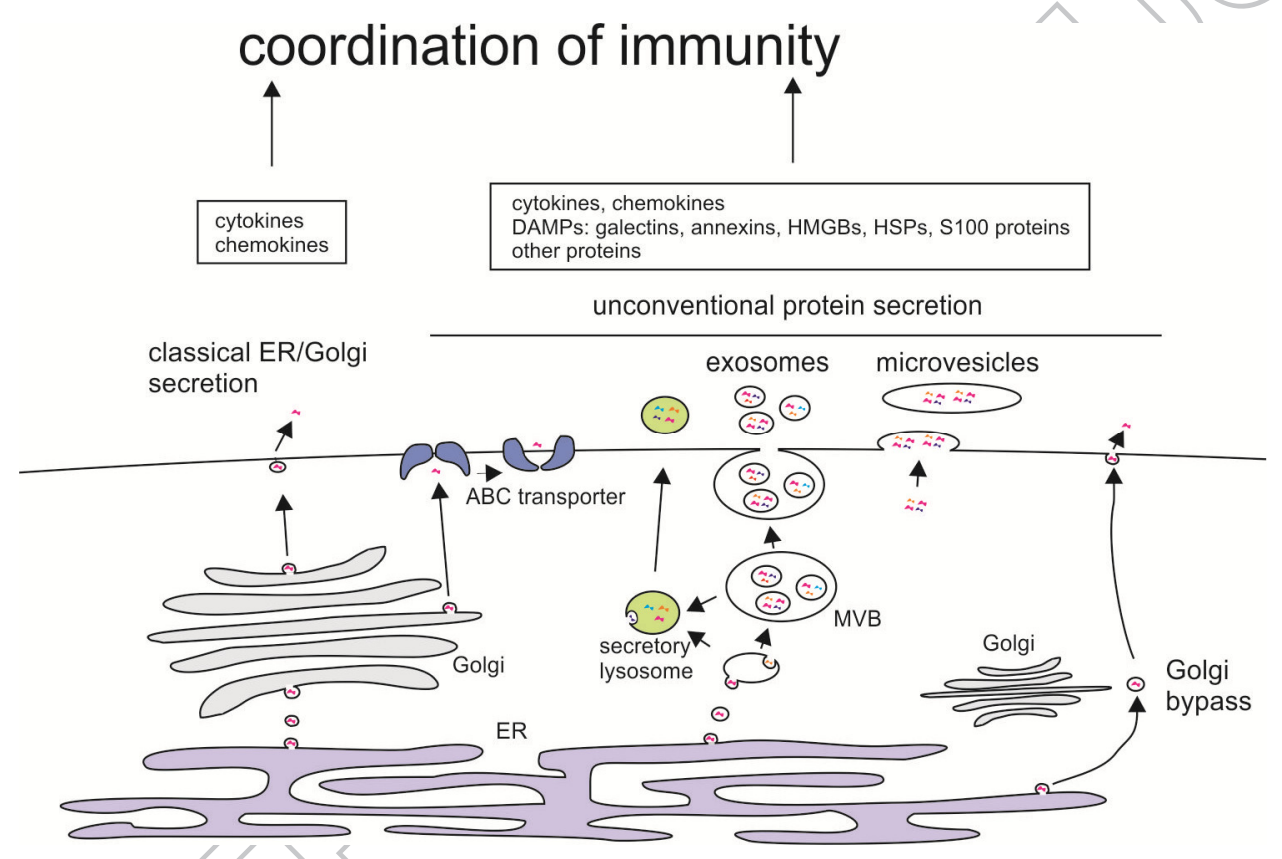


Table 1. Key findings obtained in immunology using different proteomics method. The proteomics methods have been reviewed in [3,4,9-11]

Inflammasomes

Proteomic method(s) used* Reference

AIM2 is a cytoplasmic DNA sensor for the

AP-MS

[26]

inflammasome

Gasdermin D is an inducer of pyroptosis in

AP-MS

[34]

response to non-canonical inflammasome

activation

Nek7 is an essential and novel component of

NLRP3 inflammasome

Dendritic cell subsets have differential

inflammasome function

AP-MS

label-free quantitative

Secretomes of immune cells

Caspase-1 is a regulator of unconventional protein secretion

proteomics

Influenza A virus infection of human

macrophages activates secretion of several

danger proteins

Highly sensitive secretome analysis from only

150000 mouse macrophages. Identification of 52

quantitative proteomics

(iTRAQ)

quantitative proteomics

[65]

(iTRAQ)

[5]

label-free quantitative

cytokines from TLR4-activated macrophage

secretomes

Dectin-1 pathway activates robust

unconventional protein secretion in human

macrophages

\section{EV-mediated protein secretion}

Biochemical and biological characterization reveals microvesicles and exosomes as

facilitators of HIV-1 infection

Identification of novel markers to characterize

heterogeneous populations of extracellular

proteomics

vesicle subtypes in human dendritic cells

Influenza A virus infection activates vesicle-

mediated protein secretion in human

quantitative proteomics

(iTRAQ)

macrophages

Non-canonical inflammasome activates vesicle-

mediated protein secretion in human

macrophages

\section{Mucosal immunology}

Analysis of protein expression in the small

label-free quantitative

intestine of patients infected with Vibrio cholerae

shows that a strong inflammatory response is

generated in the gut mucosa early after onset of

the infection

Altered intestinal microbiota-host mitochondrial

interaction in new onset Crohn's disease

quantitative proteomics

[73]

Immunopeptidomes

protein identification by LC-

MS/MS

label-free quantitative

proteomics

GeLC-MS/MS

[60]

GeLC-MS/MS combined with

[67]

label-free quantitative

proteomics

proteomics

(superSILAC) 
Identification of celiac disease-relevant $T$ cell epitopes. The approach presented is relevant for epitope identification in other MHC class IIassociated disorders Investigation of the bronchoalveolar lavage cells immunopeptidome from individual patients and healthy controls in order to identify diseaseassociated peptides

Large fraction of HLA class I ligands are proteasome-generated spliced peptides which could be useful in vaccine or cancer immunotherapy development

\section{$T$ cells}

GIMAP family proteins 1 and 4 are differentially regulated during human $T$ helper cell differentiation

Gut-derived Th1 and Th1/Th17 clones have major differences in the expression of cytotoxic proteins

Detailed map of the cytotoxic T lymphocyte (CTL) proteome and the effect of the metabolic checkpoint kinase mTORC1 on CTLs. Also shows how $\mathrm{mTOR}$ inhibitors control $\mathrm{T}$ cell function and program $T$ cell signal-transduction pathways

IL-2 signaling is both JAK-kinase-dependent and independent in CD8-positive T cells

CD5 transmembrane receptor constitutes a key scaffold for E3 ubiquitin-protein ligases following $T$ cell receptor stimulation
MALDI-TOF and nano-LC-

MS/MS analysis to identify

MHC peptides

optimized AP-MS to identify

[78] HLA-DR-bound peptides from low cell numbers

two-dimensional peptide prefractionation strategy followed by MS analysis

quantitative proteomics

(ICAT)

label-free quantitative

proteomics

[88]

proteomics

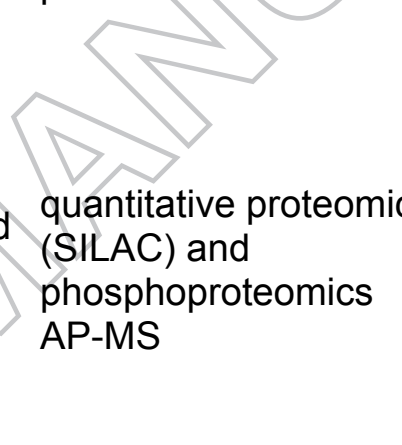

${ }^{*}$ AP-MS = affinity purification combined with mass spectrometry to identify protein complexes

iTRAQ $=$ isobaric tags for relative and absolute quantification

GeLC-MS/MS = protein separation by SDS-PAGE and identification by MS

SILAC = stable isotope labeling in cell culture

ICAT $=$ isotope-coded affinity tags 


\section{References}

Papers of special note have been highlighted as:

* of interest

${ }^{* *}$ of considerable interest

1. Broz P, Dixit VM. Inflammasomes: mechanism of assembly, regulation and signalling. Nat Rev Immunol, 16(7), 407-420 (2016).

2. Uhlén M, Fagerberg L, Hallström BM et al. Tissue-based map of the human proteome. Science, 347(6220) (2015).

3. Larance M, Lamond Al. Multidimensional proteomics for cell biology. Nat Rev Mol Cell Biol, 16(5), 269-280 (2015).

4. Aebersold R, Mann M. Mass-spectrometric exploration of proteome structure and function. Nature, 537(7620), 347-355 (2016).

5. Lietzen N, Ohman T, Rintahaka J et al. Quantitative subcellular proteome and secretome profiling of influenza A virus-infected human primary macrophages. PLoS Pathog, 7(5), e1001340 (2011).

6. Horner SM, Wilkins C, Badil S, Iskarpatyoti J, Gale M, Jr. Proteomic analysis of mitochondrial-associated ER membranes (MAM) during RNA virus infection reveals dynamic changes in protein and organelle trafficking. PLoS One, 10(3), e0117963 (2015).

7. Dill BD, Gierlinski M, Hartlova A et al. Quantitative proteome analysis of temporally resolved phagosomes following uptake via key phagocytic receptors. Mol Cell Proteomics, 14(5), 1334-1349 (2015). 
8. Naujoks J, Tabeling C, Dill BD et al. IFNs Modify the Proteome of LegionellaContaining Vacuoles and Restrict Infection Via IRG1-Derived Itaconic Acid. PLoS Pathog, 12(2), e1005408 (2016).

9. Shi T, Song E, Nie S et al. Advances in targeted proteomics and applications to biomedical research. Proteomics, 16(15-16), 2160-2182 (2016).

10. von Stechow L, Francavilla C, Olsen JV. Recent findings and technological advances in phosphoproteomics for cells and tissues. Expert Review of Proteomics, 12(5), 469-487 (2015).

11. Leitner A. Enrichment Strategies in Phosphoproteomics. In: PhosphoProteomics: Methods and Protocols. von Stechow, L (Ed. (Springer New York, New York, NY, 2016) 105-121.

12. Ohman T, Soderholm S, Paidikondala M, Lietzen N, Matikainen S, Nyman TA. Phosphoproteome characterization reveals that Sendai virus infection activates mTOR signaling in human epithelial cells. Proteomics, 15(12), 20872097 (2015).

13. Soderholm S, Kainoy DE, Ohman T et al. Phosphoproteomics to Characterize Host Response During Influenza A Virus Infection of Human Macrophages. Mol CellProteomics, 15(10), 3203-3219 (2016). *Phosphoproteome characterization of human primary macrophages combined with extensive bioinformatics and functional studies shows that cyclindependent kinase inhibitors represent potential therapeutic targets for more effective treatment of influenza infections

14. Li R, Liao G, Nirujogi RS et al. Phosphoproteomic Profiling Reveals EpsteinBarr Virus Protein Kinase Integration of DNA Damage Response and Mitotic Signaling. PLoS Pathog, 11(12), e1005346 (2015). 
15. Ye J, Zhang H, He W et al. Quantitative phosphoproteomic analysis identifies the critical role of JNK1 in neuroinflammation induced by Japanese encephalitis virus. Sci Signal, 9(448), ra98 (2016).

16. Zhang H, Sun J, Ye J et al. Quantitative Label-Free Phosphoproteomics Reveals Differentially Regulated Protein Phosphorylation Involved in West Nile Virus-Induced Host Inflammatory Response. J Proteome Res, 14(12), 51575168 (2015).

17. Lee EY, Lee HC, Kim HK et al. Infection-specific phosphorylation of glutamylprolyl tRNA synthetase induces antiviral immunity. Nat Immunol, 17(11), 12521262 (2016).

18. Liu S, Cai X, Wu J et al. Phosphorylation of innate immune adaptor proteins MAVS, STING, and TRIF induces IRF3 activation. Science, 347(6227), aaa2630 (2015).

19. Yang J, Wagner SA, Beli P. Illuminating Spatial and Temporal Organization of Protein Interaction Networks by Mass Spectrometry-Based Proteomics. Front Genet, 6, 344 (2015).

20. van Zuylen WJ, Doyon P, Clement JF et al. Proteomic profiling of the TRAF3 interactome network reveals a new role for the ER-to-Golgi transport compartments in innate immunity. PLoS Pathog, 8(7), e1002747 (2012).

21. Ohman T, Soderholm S, Hintsanen P et al. Phosphoproteomics combined with quantitative 14-3-3-affinity capture identifies SIRT1 and RAI as novel regulators of cytosolic double-stranded RNA recognition pathway. Mol Cell Proteomics, 13(10), 2604-2617 (2014). 
22. Lei $\mathrm{Y}$, Wen $\mathrm{H}, \mathrm{Yu} \mathrm{Y}$ et al. The mitochondrial proteins NLRX1 and TUFM form a complex that regulates type I interferon and autophagy. Immunity, 36(6), 933946 (2012).

23. Wertz IE, Newton K, Seshasayee D et al. Phosphorylation and linear ubiquitin direct A20 inhibition of inflammation. Nature, 528(7582), 370-375 (2015).

24. Li Y, Wang Y, Zou L et al. Analysis of the Rab GTPase Interactome in Dendritic Cells Reveals Anti-microbial Functions of the Rab32 Complex in Bacterial Containment. Immunity, 44(2), 422-437 (2016).

25. He Y, Hara H, Nunez G. Mechanism and Regulation of NLRP3 Inflammasome Activation. Trends Biochem Sci, 41(12), 1012-1021 (2016).

26. Burckstummer T, Baumann C, Bluml S et al. An orthogonal proteomicgenomic screen identifies AIM2 as a cytoplasmic DNA sensor for the inflammasome. Nat Immunol, 10(3), 266-272 (2009).

27. Wang LJ, Hsu CW, Chen CC et al. Interactome-wide analysis identifies endbinding protein 1 as a crucial component for the speck-like particle formation of activated absence in melanoma 2 (AIM2) inflammasomes. Mol Cell Proteomics, 11(11), 1230-1244 (2012).

28. He Y, Zeng MY, Yang D, Motro B, Nunez G. NEK7 is an essential mediator of NLRP3 activation downstream of potassium efflux. Nature, 530(7590), 354357 (2016). *AP-MS analysis revealed NEK7 as a major interacting partner of NLRP3 after inflammasome activation

29. Kanneganti T-D, Ozoren N, Body-Malapel M et al. Bacterial RNA and small antiviral compounds activate caspase-1 through cryopyrin/Nalp3. Nature, 440(7081), 233-236 (2006). 
30. Gross CJ, Mishra R, Schneider KS et al. K+ Efflux-Independent NLRP3 Inflammasome Activation by Small Molecules Targeting Mitochondria. Immunity, 45(4), 761-773 (2016).

31. Yan $\mathrm{Y}$, Jiang W, Liu L et al. Dopamine controls systemic inflammation through inhibition of NLRP3 inflammasome. Cell, 160(1-2), 62-73 (2015).

32. Kayagaki N, Warming S, Lamkanfi M et al. Non-canonical inflammasome activation targets caspase-11. Nature, 479(7371), 117-121 (2011).

33. Yang J, Zhao Y, Shao F. Non-canonical activation of inflammatory caspases by cytosolic LPS in innate immunity. Curr Opin Immunol, 32, 78-83 (2015).

34. He WT, Wan H, Hu L et al. Gasdermin D is an executor of pyroptosis and required for interleukin-1beta secretion. Cell Res, 25(12), 1285-1298 (2015).

35. Worah K, Mathan TS, Vu Manh TP et al. Proteomics of Human Dendritic Cell Subsets Reveals Subset-Specific Surface Markers and Differential Inflammasome Function. Cell Rep, 16(11), 2953-2966 (2016).

36. Szabo G, Dolganiuc A. The Role of Plasmacytoid Dendritic Cell-Derived IFN\&\#945; in Antiviral Immunity. 28(1), 61-94 (2008).

37. Meissner F, Scheltema RA, Mollenkopf HJ, Mann M. Direct proteomic quantification of the secretome of activated immune cells. Science, 340(6131), $475-478$ (2013).

38. Miettinen JJ, Matikainen S, Nyman TA. Global secretome characterization of herpes simplex virus 1-infected human primary macrophages. J Virol, 86(23), 12770-12778 (2012).

39. Valimaki E, Cypryk W, Virkanen J et al. Calpain Activity Is Essential for ATPDriven Unconventional Vesicle-Mediated Protein Secretion and Inflammasome Activation in Human Macrophages. J Immunol, 197(8), 3315-3325 (2016). 
40. Valimaki E, Miettinen JJ, Lietzen N, Matikainen S, Nyman TA. Monosodium urate activates Src/Pyk2/PI3 kinase and cathepsin dependent unconventional protein secretion from human primary macrophages. Mol Cell Proteomics, 12(3), 749-763 (2013).

41. Ohman T, Teirila L, Lahesmaa-Korpinen AM et al. Dectin-1 pathway activates robust autophagy-dependent unconventional protein secretion in human macrophages. J Immunol, 192(12), 5952-5962 (2014). *Combined transcriptome and global secretome analysis shows that the dectin-1 pathway induces significant gene expression changes and robust caspase-1-dependent vesicle-mediated protein secretion in human macrophages

42. Keerthikumar S, Chisanga D, Ariyaratne D et al. ExoCarta: A Web-Based Compendium of Exosomal Cargo. J Mol Biol, 428(4), 688-692 (2016).

43. Strobel M, Pfortner $\mathrm{H}$, Tuchscherr L et al. Post-invasion events after infection with Staphylococcus aureus are strongly dependent on both the host cell type and the infecting S. aureus strain. Clin Microbiol Infect, 22(9), 799-809 (2016).

44. Uhlmann J, Siemens N, Kai-Larsen Y et al. Phosphoglycerate kinase - a novel streptococcal factor involved in neutrophil activation and degranulation. $J$ Infect Dis, (2016).

45. Kwon OK, Lee W, Kim SJ et al. In-depth proteomics approach of secretome to identify novel biomarker for sepsis in LPS-stimulated endothelial cells. Electrophoresis, 36(23), 2851-2858 (2015).

46. Lee MJ, Kim J, Kim MY et al. Proteomic analysis of tumor necrosis factoralpha-induced secretome of human adipose tissue-derived mesenchymal stem cells. J Proteome Res, 9(4), 1754-1762 (2010). 
47. Oh DY, Dowling DJ, Ahmed S et al. Adjuvant-induced Human Monocyte Secretome Profiles Reveal Adjuvant- and Age-specific Protein Signatures. Mol Cell Proteomics, 15(6), 1877-1894 (2016).

48. Gyorgy B, Szabo TG, Pasztoi M et al. Membrane vesicles, current state-ofthe-art: emerging role of extracellular vesicles. Cell Mol Life Sci, 68(16), 26672688 (2011).

49. Robbins PD, Morelli AE. Regulation of immune responses by extracellular vesicles. Nat Rev Immunol, 14(3), 195-208 (2014).

50. Groot Kormelink T, Arkesteijn GJ, van de Lest CH et al. Mast Cell Degranulation Is Accompanied by the Release of a Selective Subset of Extracellular Vesicles That Contain Mast Cell-Specific Proteases. J Immunol, 197(8), 3382-3392 (2016).

51. Niu C, Wang X, Zhao M et al. Macrophage Foam Cell-Derived Extracellular Vesicles Promote Vascular Smooth Muscle Cell Migration and Adhesion. J Am Heart Assoc, 5(10) (2016).

52. Greening DW, Xu R, Gopal SK, Rai A, Simpson RJ. Proteomic insights into extracellular vesicle biology - defining exosomes and shed microvesicles. Expert Rev Proteomics, 1-27 (2016).

53. Kowal J, Arras G, Colombo M et al. Proteomic comparison defines novel markers to characterize heterogeneous populations of extracellular vesicle subtypes. Proc. Natl. Acad. Sci. U. S. A., 113(8), E968-977 (2016).

*Extensive quantitative proteomic analysis of different EV subpopulations from human dendritic cells provides guidelines to define subtypes of EVs 
54. Gould SJ, Booth AM, Hildreth JE. The Trojan exosome hypothesis. Proc. Natl. Acad. Sci. U. S. A., 100(19), 10592-10597 (2003).

55. Li M, Aliotta JM, Asara JM et al. Quantitative proteomic analysis of exosomes from HIV-1-infected lymphocytic cells. Proteomics, 12(13), 2203-2211 (2012).

56. Kadiu I, Narayanasamy P, Dash PK, Zhang W, Gendelman HE. Biochemical and biologic characterization of exosomes and microvesicles as facilitators of HIV-1 infection in macrophages. J Immunol, 189(2), 744-754 (2012).

57. Meckes DG, Jr., Gunawardena HP, Dekroon RM et al. Modulation of B-cell exosome proteins by gamma herpesvirus infection. Proc. Natl. Acad. Sci. U. S. A., 110(31), E2925-2933 (2013).

58. Zhao X, Wu Y, Duan J et al. Quantitative proteomic analysis of exosome protein content changes induced by hepatitis B virus in Huh-7 cells using SILAC labeling and LC-MS/MS. J Rroteome Res, 13(12), 5391-5402 (2014).

59. Jaworski E, Narayanan A, Van Duyne R et al. Human T-lymphotropic virus type 1 -infected cells secrete exosomes that contain Tax protein. J Biol Chem, 289(32), 22284-22305(2014).

60. Cypryk W, Lorey MB, Puustinen A, Nyman TA, Matikainen S. Proteomic and bioinformatic characterization of extracellular vesicles released from human macrophages upon influenza A virus infection. J Proteome Res, (2016).

61. Hare NJ, Chan B, Chan E, Kaufman KL, Britton WJ, Saunders BM. Microparticles released from Mycobacterium tuberculosis-infected human macrophages contain increased levels of the type I interferon inducible proteins including ISG15. Proteomics, 15(17), 3020-3029 (2015). 
62. Wang JJ, Chen C, Xie PF, Pan Y, Tan YH, Tang LJ. Proteomic analysis and immune properties of exosomes released by macrophages infected with Mycobacterium avium. Microbes Infect, 16(4), 283-291 (2014).

63. Cypryk W, Ohman T, Eskelinen EL, Matikainen S, Nyman TA. Quantitative proteomics of extracellular vesicles released from human monocyte-derived macrophages upon beta-glucan stimulation. J Proteome Res, 13(5), 24682477 (2014).

64. Reales-Calderon JA, Vaz C, Monteoliva L, Molero G, Gil C. Candida albicans Modifies the Protein Composition and Size Distribution of THP1 macrophagesderived Extracellular Vesicles. J Proteome Res, (2016).

65. Keller M, Rüegg A, Werner S, Beer H-D. Active Caspase-1 Is a Regulator of Unconventional Protein Secretion. Cell, 132(5), 818-831 (2008).

66. Zhang Y, Liu F, Yuan Y et al. Inflammasome-Derived Exosomes Activate NFkappaB Signaling in Macrophages. J Proteome Res, (2016).

67. Lorey MB, Rossi K, Eklund KK, Nyman TA, Matikainen S. Global characterization of protein secretion from human macrophages following noncanonical caspase-4/5 inflammasome activation. Molecular \& Cellular Proteomics, (2017).

68. Ellis CN, LaRocque RC, Uddin T et al. Comparative Proteomic Analysis Reveals Activation of Mucosal Innate Immune Signaling Pathways during Cholera. Infection and Immunity, 83(3), 1089-1103 (2015).

69. Starr AE, Deeke SA, Ning Z et al. Proteomic analysis of ascending colon biopsies from a paediatric inflammatory bowel disease inception cohort identifies protein biomarkers that differentiate Crohn's disease from UC. Gut, (2016). 
70. Rukmangadachar LA, Makharia GK, Mishra A et al. Proteome analysis of the macroscopically affected colonic mucosa of Crohn's disease and intestinal tuberculosis. Scientific Reports, 6, 23162 (2016).

71. Bennike TB, Carlsen TG, Ellingsen T et al. Neutrophil Extracellular Traps in Ulcerative Colitis: A Proteome Analysis of Intestinal Biopsies. Inflammatory Bowel Diseases, 21(9), 2052-2067 (2015).

72. Zhou Z, Liu H, Gu G et al. Immunoproteomic to Identify Antigens in the Intestinal Mucosa of Crohn's Disease Patients. PLoS One, 8(12), e81662 (2013).

73. Mottawea W, Chiang C-K, Mühlbauer M et al. Altered intestinal microbiotahost mitochondria crosstalk in new onset Crohn's disease. Nature Communications, 7, 13419 (2016).

74. Caron E, Kowalewski DJ, Chiek Koh C, Sturm T, Schuster H, Aebersold R. Analysis of Major Histocompatibility Complex (MHC) Immunopeptidomes Using Mass Spectrometry. Mol Cell Proteomics, 14(12), 3105-3117 (2015).

75. Dorum S, Bodd M, Fallang LE et al. HLA-DQ molecules as affinity matrix for identification of gluten T cell epitopes. J Immunol, 193(9), 4497-4506 (2014).

76. Bergseng E, Dorum S, Arntzen MO et al. Different binding motifs of the celiac disease-associated HLA molecules DQ2.5, DQ2.2, and DQ7.5 revealed by relative quantitative proteomics of endogenous peptide repertoires. Immunogenetics, 67(2), 73-84 (2015).

77. Nyambura LW, Jarmalavicius S, Baleeiro RB, Walden P. Diverse HLA-I Peptide Repertoires of the APC Lines MUTZ3-Derived Immature and Mature Dendritic Cells and THP1-Derived Macrophages. J Immunol, 197(6), 21022109 (2016). 
78. Heyder T, Kohler M, Tarasova NK et al. Approach for Identifying Human Leukocyte Antigen (HLA)-DR Bound Peptides from Scarce Clinical Samples. Mol Cell Proteomics, 15(9), 3017-3029 (2016).

79. Bassani-Sternberg M, Pletscher-Frankild S, Jensen LJ, Mann M. Mass spectrometry of human leukocyte antigen class I peptidomes reveals strong effects of protein abundance and turnover on antigen presentation. Mol Cell Proteomics, 14(3), 658-673 (2015).

80. Marino F, Mommen GP, Jeko A et al. Arginine (Di)methylated Human Leukocyte Antigen Class I Peptides Are Favorably Presented by HLA-B*07. J Proteome Res, (2016).

81. Alpízar A, Marino F, Ramos-Fernández A et al. A Molecular Basis for the Presentation of Phosphorylated Peptides by HLA-B Antigens. Molecular \& Cellular Proteomics, 16(2), 181-193 (2017).

82. Bassani-Sternberg M, Braunlein E, Klar R et al. Direct identification of clinically relevant neoepitopes presented on native human melanoma tissue by mass spectrometry. Nat Commun, 7, 13404 (2016).

83. Liepe J, Marino F, Sidney J et al. A large fraction of HLA class I ligands are proteasome-generated spliced peptides. Science, 354(6310), 354-358 (2016).

**Advanced MS-based analytical strategy shows that a large fraction of peptides bound to class I MHC on multiple human cell types are spliced together by the proteasome from two different fragments of the same protein

84. Filen JJ, Nyman TA, Korhonen J, Goodlett DR, Lahesmaa R. Characterization of microsomal fraction proteome in human lymphoblasts reveals the downregulation of galectin-1 by interleukin-12. Proteomics, 5(18), 4719-4732 (2005). 
85. Filen JJ, Filen S, Moulder R et al. Quantitative proteomics reveals GIMAP family proteins 1 and 4 to be differentially regulated during human T helper cell differentiation. Mol Cell Proteomics, 8(1), 32-44 (2009).

86. Moulder R, Lonnberg T, Elo LL et al. Quantitative proteomics analysis of the nuclear fraction of human CD4+ cells in the early phases of IL-4-induced Th2 differentiation. Mol Cell Proteomics, 9(9), 1937-1953 (2010).

87. Riaz T, Sollid LM, Olsen I, de Souza GA. Quantitative Proteomics of GutDerived Th1 and Th1/Th17 Clones Reveal the Presence of CD28+ NKG2DTh1 Cytotoxic CD4+ T cells. Mol Cell Proteomics, 15(3), 1007-1016 (2016).

88. Hukelmann JL, Anderson KE, Sinclair LV et al. The cytotoxic T cell proteome and its shaping by the kinase mTOR. Nat Immunol, 17(1), 104-112 (2016). *A label-free quantitative proteome analysis provides comprehensive understanding of cytotoxic T lymphocyte $(C T L)$ identity and the control of CTL function by mTORC1

89. Bottcher JP, Beyer M, Meissner F et al. Functional classification of memory CD8(+) T cells by CX3CR1 expression. Nat Commun, 6, 8306 (2015).

90. Navarro MN, Feijoo-Carnero C, Arandilla AG, Trost M, Cantrell DA. Protein kinase D2 is a digital amplifier of T cell receptor-stimulated diacylglycerol signaling in naive CD8(+) T cells. Sci Signal, 7(348), ra99 (2014).

91. Osinalde N, Moss H, Arrizabalaga O et al. Interleukin-2 signaling pathway analysis by quantitative phosphoproteomics. J Proteomics, 75(1), 177-191 (2011).

92. Osinalde N, Sanchez-Quiles V, Akimov V, Blagoev B, Kratchmarova I. SILACbased quantification of changes in protein tyrosine phosphorylation induced by Interleukin-2 (IL-2) and IL-15 in T-lymphocytes. Data Brief, 5, 53-58 (2015). 
93. Osinalde N, Sanchez-Quiles V, Akimov V, Guerra B, Blagoev B, Kratchmarova I. Simultaneous dissection and comparison of IL-2 and IL-15 signaling pathways by global quantitative phosphoproteomics. Proteomics, 15(2-3), 520$531(2015)$.

94. Osinalde N, Mitxelena J, Sanchez-Quiles V et al. Nuclear Phosphoproteomic Screen Uncovers ACLY as Mediator of IL-2-induced Proliferation of CD4+ T lymphocytes. Mol Cell Proteomics, 15(6), 2076-2092 (2016).

95. Osinalde N, Sanchez-Quiles V, Akimov V et al. Characterization of ReceptorAssociated Protein Complex Assembly in Interleukin (IL)-2- and IL-15Activated T-Cell Lines. J Proteome Res, (2016).

96. Osinalde N, Sanchez-Quiles V, Blagoev B, Kratchmarova I. Changes in Gab2 phosphorylation and interaction partners in response to interleukin (IL)-2 stimulation in T-lymphocytes. Sci Rep, 6, 23530 (2016).

97. Ross SH, Rollings C, Anderson KE, Hawkins PT, Stephens LR, Cantrell DA. Phosphoproteomic Analyses of Interleukin 2 Signaling Reveal Integrated JAK Kinase-Dependent and -Independent Networks in CD8(+) T Cells. Immunity, 45(3), 685-700 (2016).

98. Navarro MN, Goebel J, Feijoo-Carnero C, Morrice N, Cantrell DA. Phosphoproteomic analysis reveals an intrinsic pathway for the regulation of histone deacetylase 7 that controls the function of cytotoxic T lymphocytes. Nat Immunol, 12(4), 352-361 (2011).

99. Voisinne G, Garcia-Blesa A, Chaoui K et al. Co-recruitment analysis of the CBL and CBLB signalosomes in primary T cells identifies CD5 as a key regulator of TCR-induced ubiquitylation. Mol Syst Biol, 12(7), 876 (2016). 
100. Roncagalli R, Cucchetti M, Jarmuzynski $\mathrm{N}$ et al. The scaffolding function of the RLTPR protein explains its essential role for CD28 co-stimulation in mouse and human T cells. J Exp Med, 213(11), 2437-2457 (2016).

101. Heaton SM, Borg NA, Dixit VM. Ubiquitin in the activation and attenuation of innate antiviral immunity. J Exp Med, 213(1), 1-13 (2016).

102. Chiang C, Gack MU. Post-translational Control of Intracellular Pathogen Sensing Pathways. Trends Immunol, (2016).

103. Szabo-Taylor K, Ryan B, Osteikoetxea X et al. Oxidative and other posttranslational modifications in extracellular vesicle biology. Semin Cell Dev Biol, 40, 8-16 (2015).

104. Guo J, Cui Y, Yan Z et al. Phosphoproteome Characterization of Human Colorectal Cancer SW620 Cell-Derived Exosomes and New Phosphosite Discovery for C-HPP. J Proteome Res, 15(11), 4060-4072 (2016).

105. Atkuri KR, Stevens JC, Neubert $H$. Mass cytometry: a highly multiplexed single-cell technology for advancing drug development. Drug Metab Dispos, 43(2), 227-233 (2015).

106. Giesen C, Wang HA, Schapiro D et al. Highly multiplexed imaging of tumor tissues with subcellular resolution by mass cytometry. Nat Methods, 11(4), $417-422(2014)$.

107. Marakalala MJ, Raju RM, Sharma K et al. Inflammatory signaling in human tuberculosis granulomas is spatially organized. Nat Med, 22(5), 531-538 (2016). ${ }^{* *}$ Combination of laser-capture microdissection, mass spectrometry and confocal microscopy generated detailed molecular maps of human granulomas. 
108. Holzlechner M, Strasser K, Zareva E et al. In Situ Characterization of TissueResident Immune Cells by MALDI Mass Spectrometry Imaging. J Proteome Res, (2016).

109. Vidal M, Chan DW, Gerstein M et al. The human proteome - a scientific opportunity for transforming diagnostics, therapeutics, and healthcare. Clin Proteomics, 9(1), 6 (2012).

110. Mann M. Can proteomics retire the western blot? J Proteome Res, 7(8), 3065 (2008).

111. Cypryk W. Extracellular vesicles in innate immunity : Proteomic investigations. (online) Available at: http://urn.fi/URN:ISBN:978-951-51-2448-7 Last accessed 11th April 2017. 